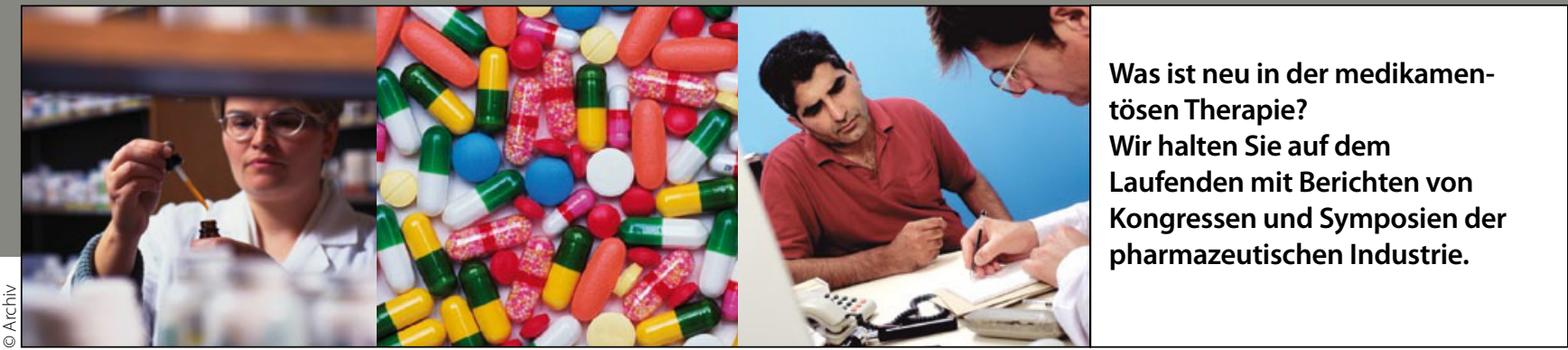

\title{
Vorhofflimmern
}

\section{Thrombin-Hemmer uniform wirksamer als Warfarin}

- Voraussichtlich Mitte des Jahres wird es eine neue Behandlungsoption für Patienten mit Vorhofflimmern (VF) geben: Statt mit Vitamin-K-Antagonisten (wie Marcumar $^{\circledast}$ ) werden sie sich mit dem neuen oralen Antikoagulans Dabigatran vor Schlaganfällen schützen können. Der als Pradaxa ${ }^{\circledR}$ bereits zur Prophylaxe von Thromboembolien nach Knie- und Hüftgelenksoperationen zugelassene direkte Thrombin-Hemmer erleichtert die Behandlung im Vergleich zu Vitamin-K-Antagonisten erheblich: Es gibt eine fixe Dosierung, eine INR-Kontrolle ist nicht notwendig und Interaktionen mit Arznei- oder Nahrungsmitteln sind deutlich seltener.

Im April wurde die Empfehlung für die Zulassung zur Schlaganfallprophylaxe bei Vorhofflimmern in Europa ausgesprochen. Diese fußt im Wesentlichen auf der Meilensteinstudie RE-LY, die bei 18000 Patienten mit Vorhofflimmern drei Antikoagulanzienstrategien verglich: Warfarin-Standardbehandlung sowie zwei Dosierungen von Dabigatran $(2 \times 110 \mathrm{mg} / \mathrm{d}$ und $2 \times 150 \mathrm{mg} / \mathrm{d})$.

\section{Hämorrhagische Schlaganfälle seltener}

Im Laufe von zwei Jahren zeigte sich: Die niedrigere Dabigatrandosierung schützte ebenso effektiv vor Schlaganfällen oder systemischen Embolien wie Warfarin ( $1,53 \%$ vs. $1,69 \%$ pro Jahr), hatte aber ein geringeres Blutungsrisiko (2,71\% vs. $3,36 \%$ pro Jahr). Die höhere Dabigatrandosierung war wirksamer als Warfarin (Schlaganfall oder systemische Embolien 1,11\% vs. $1,69 \%)$, das Blutungsrisiko war gleich $(3,11 \%$ vs. $3,36 \%$ pro Jahr). Hämorrhagische Schlaganfälle waren unter beiden Dabigatrandosierungen seltener. Bei der Mortalität zeigten sich keine Unterschiede.
Auf dem Kongress des American College of Cardiology wurden neue Substudien vorgestellt. Die Art des Vorhofflimmerns - paroxysmal, persistierend, permanent - hatte keinen Einfluss auf die Ergebnisse. Dabigatran 2 x $150 \mathrm{mg} / \mathrm{d}$ war bei allen drei VF-Typen wirksamer als Warfarin. Die niedrigere Dabigatrandosis war bei allen drei VF-Typen sicherer.

In der zweiten Substudie analysierte man, ob die Höhe des Schlaganfallrisikos einen Einfluss auf das Therapieergebnis hatte. Dazu bediente man sich des von den EU-Leitlinien empfohlenen $\mathrm{CHA}_{2} \mathrm{DS}_{2}$ VAScRisiko-Scores. Auch hier zeigte sich, dass Dabigatran 2 x 150 mg/d in allen Risikogruppen Warfarin überlegen war.

\section{- Dr. Dirk Einecke}

Quelle: Jahrestagung des American College of Cardiology, New Orleans, April 2011 (unterstützt von Boehringer Ingelheim)

\section{Die wichtigsten Ergebnisse der RE-LY-Substudien}

MMW: Welche Botschaft entnehmen Sie den neuen Substudien von RE-LY?

Böhm: Dabigatran ist über das gesamte Risikospektrum einer Warfarinbehandlung überlegen: Von einem niedrigen $\mathrm{CHA}_{2} \mathrm{DS}_{2}$ VASc-Score von 0 bis 2 bis hin zu einem sehr hohen Risiko für Schlaganfälle, entsprechend einem Score von 5 bis 9. Es gab auch bezüglich des Blutungsrisikos keinen Unterschied.

MMW: Bei welchen Patienten mit Vorhofflimmern würden Sie Dabigatran einsetzen? Böhm: Bei Neueinstellungen ziehe ich die Substanz vor, weil sie eindeutig Überlegenheit bewiesen hat oder in der niedrigeren
Dosierung weniger Blutungskomplikationen verursacht. Die therapeutische Breite, also der Abstand zwischen Nutzen und Risiko, ist größer geworden im Vergleich zu Vitamin-K-Antagonisten.

Patienten, die über Jahre mit Marcumar gut eingestellt sind, würde ich nicht umstellen. Doch viele dieser Patienten sind Marcumar gegenüber abgeneigt, sodass sie die neue Therapie bevorzugen werden. Einen entscheidenden Fortschritt bedeutet Dabigatran für Patienten mit Vorhofflimmern, die eine Antikoagulation benötigen, bisher aber aufgrund von Kontraindikationen oder Angst vor Blutungen kein Marcumar nehmen.

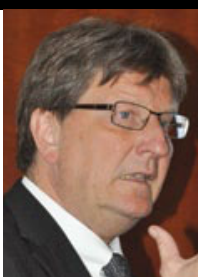 \\ Nachgefragt bei Prof. Dr. Michael Böhm \\ Universität Homburg/ Saar}

MMW: Der Arzt hat zwei Dosierungsoptionen: wann nimmt man welche? Böhm: Beide Dosierungen haben ihre Berechtigung. Bei Patienten mit Blutungsrisiken oder solchen, die bereits Blutungen hatten, wäh/t man die sicherere Dosierung, in anderen Fällen die wirksamere.

- Interview: Dr. Dirk Einecke 